\title{
EMBOLIZAÇÃO DE EMERGÊNCIA EM ADENOMA HEPÁTICO ROTO
}

\section{EMERGENCY TRANSARTERIAL EMBOLIZATION IN RUPTURED HEPATOCELLULAR ADENOMA}

Valéria Catharina Theodorovitz ${ }^{1}$; Beatriz Sordi Chara ${ }^{1}$; Gabriela Baum ${ }^{1}$; Claudia Theis ${ }^{2}$; Karina Luiza Zimmermann²; Murilo Pilatti ${ }^{1,2}$.

\section{RESUMO}

Introdução: O adenoma hepatocelular (AHC) consiste em uma neoplasia hepática benigna que tem como principal fator de risco o uso prolongado de contraceptivos orais a base de estrogênio. A suspeita diagnóstica costuma ser feita através da presença de sintomas de abdome agudo hemorrágico. Técnicas minimamente invasivas vêm sendo cada vez mais propostas para diminuir a morbimortalidade. Relato do caso: Este artigo expõe o caso de uma paciente jovem do sexo feminino, hemodinamicamente instável e diagnosticada com grande AHC roto. Conclusão: A paciente foi submetida a tratamento por meio da embolização transarterial (ETA), ainda na fase aguda, resultando em controle do sangramento, estabilização do quadro e sucesso terapêutico.

Palavras chave: Adenoma de Células Hepáticas. Embolização Terapêutica. Medicina de Emergência.

\section{ABSTRACT}

Introduction: Hepatocellular adenoma (HCA) is a neoplastic liver lesion strongly associated with a long term use of Estrogen Oral Contraceptive Pill (EOCP). Diagnoses are most often made during symptoms of hemorrhagic acute abdomen. Minimally-invasive techniques has been recommended in order to decrease morbidity and mortality. Case report: This article reports a case of large HCA ruptured, diagnosed in a young female whom arrived hemodynamically instable at emergency room and was treated by Transarterial Embolization (TEA). Conclusion: The treatment controlled the acute bleeding and successfully stabilizes the patient.

Keywords: Adenoma, Liver Cell. Embolization, Therapeutic. Emergency Medicine.

\section{INTRODUÇÃO}

O Adenoma Hepático ou Hepatocelular (AHC) é uma neoplasia hepática rara proveniente do tecido epitelial, cuja prevalência vem aumentando ao longo das últimas décadas principalmente em mulheres na fase reprodutiva. $O$ aumento de sua incidência foi atribuído a difusão do uso de pílulas anticoncepcionais orais (ACO), além de avanços e maior disponibilização das técnicas de diagnóstico por imagem ${ }^{1-3}$. A associação da patologia com a utilização de ACO contendo estrogênio teve sua descrição inicial em 1973, quando foram relatados os primeiros sete casos. $\mathrm{Na}$ atualidade, estima-se que a cada ano, cerca de $3 \mathrm{em}$ cada 100.000 mulheres que utilizam ACO a longo prazo, desenvolvem AHC4-6.

As orientações para manejo do AHC também sofreram algumas mudanças ao longo dos últimos anos, atualmente, a conduta é definida pelo estado hemodinâmico do paciente. Diante de quadros estáveis, pode ser realizado tratamento conservador, com acompanhamento da evolução por meio de exames de imagem, e em condições de instabilidade persistente ou hemoperitônio, maiores intervenções são indicadas ${ }^{4,5}$. Nesses casos, técnicas minimamente invasivas, como a Embolização Transarterial (ETA), vêm sendo empregadas por estarem associadas a menor índice de letalidade e melhor prognóstico quando comparado às ressecções cirúrgicas ${ }^{1-4}$.

\section{RELATO DO CASO}

Paciente feminina, 31 anos, previamente hígida, obesa, com índice de massa corporal (IMC) de 37 e história de uso de contraceptivo oral desde os 14 anos de idade, deu entrada no Hospital Municipal São José (HMSJ) de Joinville (SC), devido a dor abdominal de início súbito, de forte intensi-

${ }^{1}$ Universidade da Região de Joinville (UNIVILLE) - Departamento de Medicina - Joinville - SC- Brasil ${ }^{2}$ Hospital Municipal São José de Joinville (HMSJ) - Departamento de Cirurgia Geral - Joinville - SC Brasil 
dade em hipocôndrio direito, com irradiação para dorso, associada a astenia. Negava história de trauma. Ao exame físico apresentava-se hipotensa e taquicárdica, com dor abdominal, porém sem sinais de irritação peritoneal. Exames laboratoriais evidenciaram Hemoglobina $(\mathrm{Hb})$ de $5 \mathrm{~g} / \mathrm{dl}$, Hematócrito (Ht) de 16,5\%, leucócitos $16.360 / \mathrm{mm}^{3}$, plaquetas $268000 / \mathrm{mm}^{3}$, Transglutaminase Oxalacética (TGO) 1960 U/L, Transglutaminase Pirúvica (TGP) 1697 U/L, Fosfatase Alcalina (FA) $627 \mathrm{U} / \mathrm{L}$, Gama Glutamil Transpeptidase (GGT) 335 U/L. A ultrassonografia (USG) de abdome realizada demonstrou volumosa tumoração sólida e heterogênea em lobo direito, medindo $18,3 \times 15,5 \times 15,5 \mathrm{~cm}$, com líquido livre na cavidade.

Prosseguiu-se investigação com Tomografia Computadorizada (TC) contrastada de abdome, a qual evidenciou formação expansiva nodular e heterogênica com conteúdo hemorrágico (Figura 1), comprometendo grande parte do lobo hepático direito, provocando o deslocamento medial das estruturas do hilo hepático e da veia cava inferior e presença de líquido livre intraperitoneal. O exame detectou extravasamento de contraste no interior da massa sugerindo sangramento ativo (Figura 2).

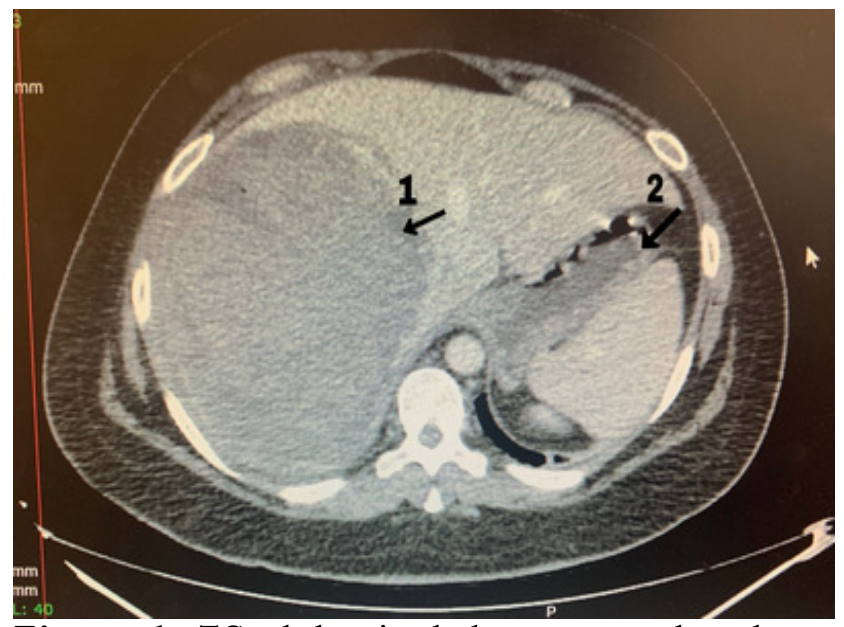

Figura 1. TC abdominal demonstrando adenoma hepatocelular roto (seta 1) e líquido livre no espaço subfrênico (seta 2).

Devido à suspeita de adenoma hepático roto, contatou-se a cirurgia vascular, que optou pela realização de embolização de emergência de segmento acometido. Após a realização da ETA a paciente evoluiu com melhora gradual de marcadores hepáticos, estabilidade de $\mathrm{HB}$ e $\mathrm{HT}$, seriados exames de imagem que não evidenciaram novos episódios de sangramento, não sendo necessária a complementação terapêutica por cirurgia aberta.

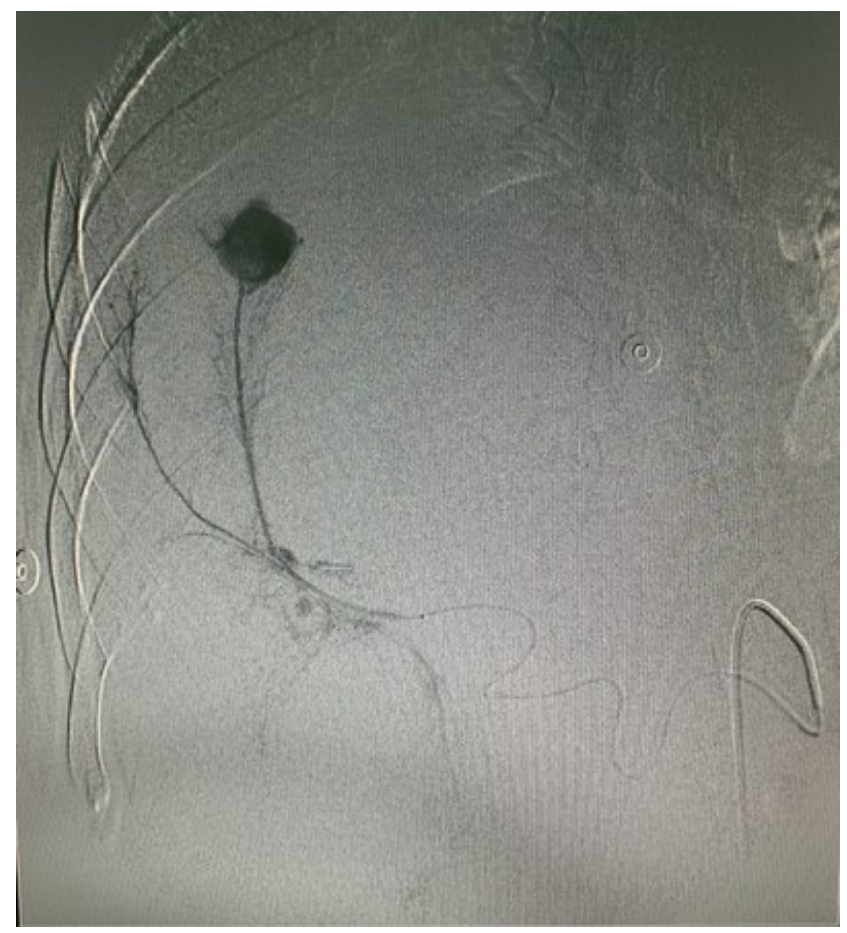

Figura 2. Arteriografia com extravasamento do contraste na massa nodular hepática (segmento 8) demonstrando sangramento ativo.

A paciente recebeu alta após duas semanas de internação, com boa evolução e acompanhamento com equipe de cirurgia hepatobiliar do HMSJ. Atualmente, permanece assintomática e realiza seguimento ambulatorial a cada três meses por meio de USG e TC abdominais, mantendo imagem compatível com adenoma hepático em lobo direito.

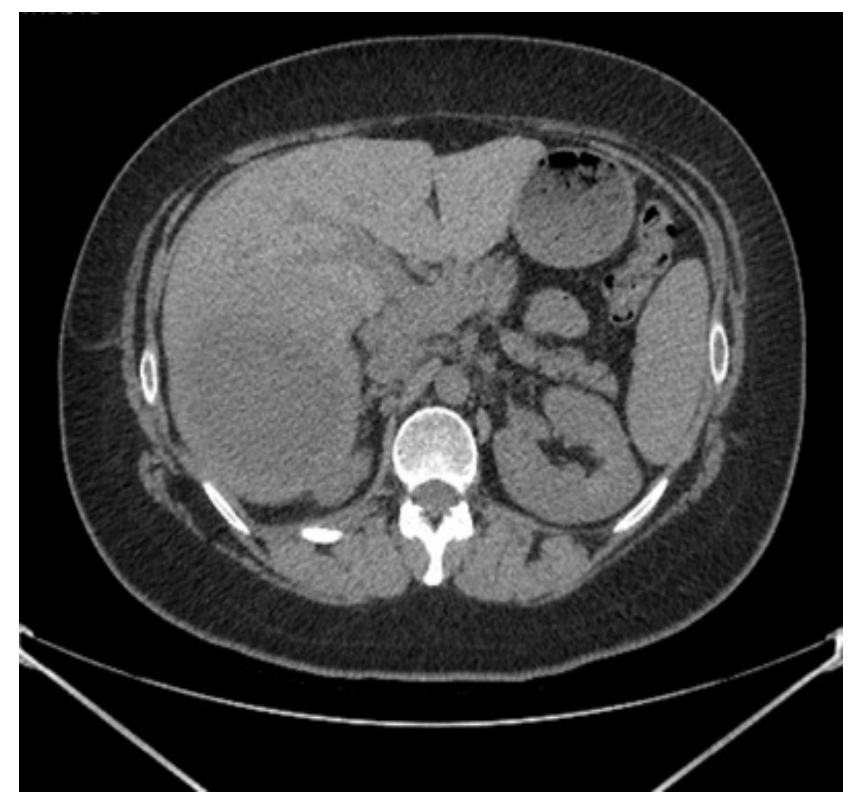

Figura 3. TC abdominal após embolização transarterial demonstrando redução do volume do AHC. 


\section{DISCUSSÃO}

O Adenoma Hepatocelular (AHC) apresenta diversos fatores de risco, como obesidade, sindrome metabólica, hipertensão e dislipidemia, sendo o uso de anticoncepcionais orais o fator de risco de maior relevância epidemiológica. A incidência anual dessa neoplasia aumentou nas últimas décadas, sendo atualmente de 1 para 1 milhão em mulheres que nunca utilizaram ACOs, e de 35 para 1 milhão por ano em pacientes que utilizam contraceptivos orais há longo prazo ${ }^{3}$.

A importância clínica desta patologia é relacionada ao seu potencial de evolução para complicações hemorrágicas e malignização, frequentemente necessitando de maiores intervenções ${ }^{4,5}$. No entanto, a sua maioria apresenta-se assintomático sendo identificado de maneira incidental através de exames de imagem ou, então, em quadros já complicados com sinais de ruptura e sangramento abdominal. Assim, podendo estar associado a dor súbita em quadrante superior direito, anorexia, vômito e febre 7 . No caso relatado, a paciente foi admitida com dor em hipocôndrio direito associada a instabilidade hemodinâmica, sendo então, submetida a exames de imagem e laboratório nos quais foi evidenciada lesão hemorrágica de foco hepático.

Em relação aos parâmetros laboratoriais, são frequentes as alterações nos valores de GGT e FA7 7 . Anormalidades nos testes de lesão hepática são mais raras, mas estavam presentes no quadro da paciente relatada, bem como altos niveis de GGT e FA.

$\mathrm{Na}$ investigação por imagem, o USG de abdome pode ser útil inicialmente, pois é capaz de identificar tumorações sólidas e tecidos de aspecto necrótico. Por outro lado, com a TC, são obtidas imagens de melhor definição, nas quais é possivel diferenciar áreas de necrose, sangramento ou fibrose, além da presença de líquido livre e lesões em expansão, como demonstrado no caso $^{7}$.

Quando identificado um adenoma sem sinais de ruptura, pode-se definir a conduta terapêutica de acordo com seu tamanho. Lesões pequenas, com menos de 5 $\mathrm{cm}$ de diâmetro, raramente desencadeiam sintomas ou complicações e podem ser tratadas de maneira conservadora. Lesões maiores que $5 \mathrm{~cm}$ e/ou quadros sintomáticos, especialmente diante de sinais de alarme para transformação maligna em exames de imagem, possuem indicação de ressecção cirúrgica eletiva ${ }^{8}$. No entanto, a utilização de terapias minimamente invasivas como a ETA tem demonstrado maior eficácia e vem ganhando espaço no tratamento do adenoma hepático, independentemente de seu tamanho inicial1,6.

Em casos de AHC rotos, o manejo é voltado à hemostasia e a garantia da estabilidade hemodinâmica por meio de ressuscitação volêmica, transfusão e embolização adequadas $^{9}$. A ressecção cirúrgica de emergência deve ser evitada pois demonstra taxas de mortalidade 5-10 vezes maior em comparação à cirurgia eletiva, entretanto, pode ser necessária em casos de instabilidade refratária10-12. Em seu lugar, a ETA, mostra-se muito mais segura e efetiva na contenção da hemorragia, além de reduzir os indices de reabordagem tardia por necrose do adenoma ${ }^{9}$.

A investigação subsequente à terapia deve ser realizada para verificar a etiologia da lesão, não devendo ser retardada. Isso porque, na presença de carcinomas hepatocelulares, adenomas atípicos ou lesões com risco de malignização a ressecção cirúrgica deve ser discutida. Já em casos de adenomas confirmados, a remoção cirúrgica tardia tem se mostrado desnecessária com o uso da ETA $^{9,12}$. Em seguimento, deve ser feita a interrupção do uso de $\mathrm{ACO}$ e o acompanhamento com exames de imagem como tomografia ou ressonância magnética. ${ }^{1}$

No caso clínico apresentado, a rápida identificação da ruptura do adenoma e opção pelo tratamento com embolização foi o que garantiu melhora hemodinâmica, permitindo sucesso terapêutico e a boa evolução da paciente, não sendo necessária nova abordagem cirúrgica para ressecção da lesão.

\section{REFERÊNCIAS}

1. Nasser F, Affonso BB, Galastri FL, Odisio BC, Garcia RG. Minimally invasive treatment of hepatic adenoma in special cases. Einstein (Sao Pau1o). 2013;11(4):524-7. doi: 10.1590/ s167945082013000400021

2. Pinto LEV, Silva JPR, Rêgo GC, Garcia JHP. Tratamento cirúrgico tardio para ruptura espontânea de adenoma hepatocelular: relato de caso. Arq Bras Cir Dig. 2015;28(4):293-301. 
doi:/ 10.1590/S01026720201500030 022 .

3. Marrero JA, Ahn J, Rajender Reddy $\mathrm{K}$; American College of Gastroenterology. ACG clinical guideline: the diagnosis and management of focal liver lesions. Am J Gastroenterol. 2014;109(9):1328-47. doi:10.1038 /ajg.2014.213

4. European Association for the Study of the Liver (EASL). EASL Clinical Practice Guidelines on the management of benign liver tumours. $\mathrm{J}$ Hepatol. 2016;65(2):386-98. doi: 10.1016/j.jhep.2016.04. 001.

5. Klompenhouwer AJ, De Man RA, Thomeer MGJ, Ijzermans JNM. Management and outcome of hepatocellular adenoma with massive bleeding at presentation. World J Gastroenterol. 2017;23(25:4579-86. doi: 10.3748/wjg.v23.i25.4579.

6. Agrawal $\mathrm{S}$, Agarwal $\mathrm{S}$, Arnason $\mathrm{T}$, Saini S, Belghiti J. Management of hepatocellular adenoma: Recent advances. Clin Gastroenterol Hepatol. 2015;13(7):1221-30. doi: 10.1016/ j.cgh.2014.05.023.

7. Vargas-Flores E, Pérez-Aguilar F, Valdez-Mendieta Y. [Extracapsular hepatic adenoma. Case report and literature review]. Cir Cir. 2017;85(2):175-80. Spanish. doi: $10.1016 /$ j.circir.2015.12.006.

8. McKay B, Webber L, Manoharan B, Miller G, Clouston A, Bryant R, et al. Large size and haemorrhage are not contraindications to laparoscopic resection of hepatocellular adenoma. ANZ J Surg. 2020;90(6):1099-103. doi: 10.1111 /ans. 15702 .
9. Dokmak S, Aussilhou B, Rasoaherinomenjanahary $\mathrm{F}$, Ronot $\mathrm{M}$, Dahdouh R, Ftériche FS, et al. Hemorrhage of hepatocellular adenoma: a complication that can be treated by conservative management without surgery.HPB. 2018;20(12):1198-205. doi: 10.1016/j.hpb.2018.06.1796.

10. Thomeer MG, Broker M, Verheij J, Doukas M, Terkivatan T, Bijdevaate $\mathrm{D}$, et al. Therap Adv Gastroenterol 2016;9(6):898-912. doi: 10.1177/ $1756283 X 16663882$.

11. Zhao C, Pei S-L, Cucchetti A, Tong TJ, Ma Y-L, Zhong J-H, et al. Systematic review: benefits and harms of transarterial embolisation for treating hepatocellular adenoma. Aliment Pharmacol Ther. 2017;46:83. doi: 10.1111/apt.14044.

12. Darnis B, Rode A, Mohkam K, Ducerf C, Mabrut JY. Management of bleeding liver tumors. J Visc Surg. 2014;151(5):365-75. doi: 10.1016/j. jviscsurg.2014.05.007.

Fonte de financiamento: Não

Conflito de interesses: Não

Data de Submissão: 06 Junho 2020

Decisão final: 08 Março 2021

\section{E-mail do autor:}

Valéria Catharina Theodorovitz

E-mail: valeria.theo@hotmail.com valeriaatheo@gmail.com 